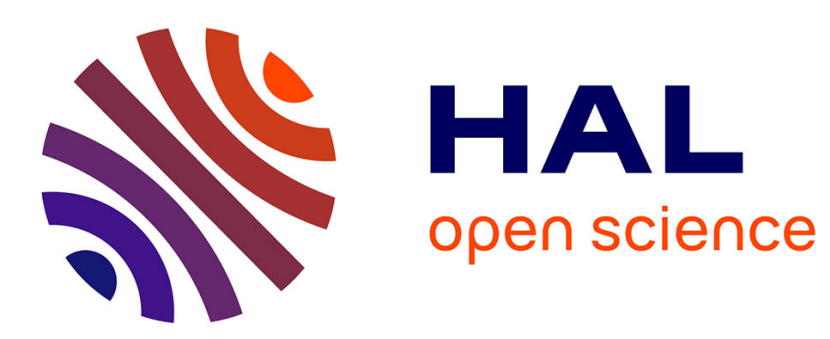

\title{
Solvability of monotone systems of fully nonlinear elliptic PDE's
}

\author{
Alexander Quaas, Boyan Sirakov
}

\section{To cite this version:}

Alexander Quaas, Boyan Sirakov. Solvability of monotone systems of fully nonlinear elliptic PDE's.

Comptes Rendus. Mathématique, 2008, 346 (11-12), pp.641-644. hal-00138111

\section{HAL Id: hal-00138111 \\ https://hal.science/hal-00138111}

Submitted on 23 Mar 2007

HAL is a multi-disciplinary open access archive for the deposit and dissemination of scientific research documents, whether they are published or not. The documents may come from teaching and research institutions in France or abroad, or from public or private research centers.
L'archive ouverte pluridisciplinaire HAL, est destinée au dépôt et à la diffusion de documents scientifiques de niveau recherche, publiés ou non, émanant des établissements d'enseignement et de recherche français ou étrangers, des laboratoires publics ou privés. 


\title{
Solvability of monotone systems of fully nonlinear elliptic PDE's
}

\author{
Alexander QUAAS ${ }^{1}$ \\ Departamento de Matemática, Universidad Santa Maria \\ Avenida España 1680, Casilla 110-V, Valparaíso, Chile \\ Boyan SIRAKOV \\ UFR SEGMI, Université Paris 10, 92001 Nanterre Cedex, France \\ and CAMS, EHESS, 54 bd Raspail, 75270 Paris Cedex 06, France
}

In this note we study quasimonotone systems of fully nonlinear equations, in the form

$$
\left\{\begin{aligned}
H_{1}\left(D^{2} u_{1}, D u_{1}, u_{1}, \ldots, u_{n}, x\right) & =f_{1}(x) \\
H_{2}\left(D^{2} u_{2}, D u_{2}, u_{1}, \ldots, u_{n}, x\right) & =f_{2}(x) \\
& \cdots \\
H_{n}\left(D^{2} u_{n}, D u_{n}, u_{1}, \ldots, u_{n}, x\right) & =f_{n}(x),
\end{aligned}\right.
$$

in a bounded domain $\Omega \subset \mathbb{R}^{N} ; n, N \geq 1$. Here $H_{i}$ are uniformly elliptic fully nonlinear operators of Isaac's type.

Our work is motivated by the well-known paper [IK], where the theory of viscosity solutions was applied to get existence and uniqueness results for this type of systems. More precisely, in that paper conditions were given under which the system satisfies a comparison principle (and so it cannot have more than one solution), and it was shown that whenever the system has a subsolution and a supersolution, which are ordered, then Perron's method guarantees the existence of a solution. It is our goal here, on one hand, to extend as far as possible the uniqueness result from [IK] in the uniformly elliptic case, that is, to give a necessary and sufficient condition under which the system satisfies a comparison principle, and on the other hand, to give explicit conditions under which ordered sub- and supersolutions can be found. For a large class of systems our results are optimal. Further, we aim at proving results of existence of eigenvalues of vector fully nonlinear operators.

We shall make essential use of the results in the two recent papers [QS], where corresponding results for scalar equations were obtained, and [BS], where an Alexandrov-Bakelman-Pucci and Harnack type estimates for systems of type (1) were proved.

\footnotetext{
${ }^{1}$ Supported by FONDECYT, Grant N. 1040794
} 
We intend to consider nonlinear operators of Isaac's type, that is, $H_{k}$ will be allowed to be a sup-inf of coupled linear operators

$$
\sup _{\alpha \in \mathcal{A}_{k}} \inf _{\beta \in \mathcal{B}_{k}}\left\{\sum_{i, j=1}^{N} a_{i j, k}^{\alpha, \beta}(x) \partial_{i j} u_{k}+\sum_{i=1}^{N} b_{i, k}^{\alpha, \beta}(x) \partial_{i} u_{k}+\sum_{j=1}^{n} c_{j, k}^{\alpha, \beta}(x) u_{j}\right\} .
$$

where $\mathcal{A}_{k}, \mathcal{B}_{k}$ are arbitrary index sets. When $\mathcal{B}_{k}=\emptyset$ in (2) the corresponding sup-operator is usually referred to as Hamilton-Jacobi-Bellman (HJB) operator. These operators are essential tools in control theory and in theory of large deviations, while Isaac's operators are basic in game theory. We refer to $[\mathrm{BS}],[\mathrm{IK}]$, and the references in these papers, for a larger list of problems, where systems of type (1) appear.

Before stating our general hypotheses on $H_{i}$ in (1), we note for the reader's convenience that they are satisfied by operators of type (2) for instance if the matrices $\left(a_{i j, k}^{\alpha, \beta}\right)$ are continuous and uniformly positive definite, and if $b_{i, k}^{\alpha, \beta}, c_{i, k}^{\alpha, \beta} \in L^{\infty}(\Omega), c_{j, k}^{\alpha, \beta} \geq 0$ for $j \neq k$, for all $\alpha, \beta, i, j, k$.

Next, we list our hypotheses on (1). We shall use the following notation. We define, for all $M, N \in \mathcal{S}_{N}, p, q \in \mathbb{R}^{N}$, the extremal operators $\mathcal{L}^{-}, \mathcal{L}^{+}$by

$$
\mathcal{L}^{-}(M, p)=\mathcal{M}_{\lambda, \Lambda}^{-}(M)-\gamma|p|, \quad \mathcal{L}^{+}(M, p)=\mathcal{M}_{\lambda, \Lambda}^{+}(M)+\gamma|p|,
$$

for some constants $0<\lambda \leq \Lambda ; \gamma \geq 0$. Here $\mathcal{M}^{+}, \mathcal{M}^{-}$denote the Pucci operators $\mathcal{M}_{\lambda, \Lambda}^{+}(M)=\sup _{A \in \mathcal{A}} \operatorname{tr}(A M), \mathcal{M}_{\lambda, \Lambda}^{-}(M)=\inf _{A \in \mathcal{A}} \operatorname{tr}(A M)$, where $\mathcal{A} \subset \mathcal{S}_{N}$ denotes the set of matrices whose eigenvalues lie in $[\lambda, \Lambda]$.

For a function $f$ we denote $f_{+}=\max \{f, 0\}, f=f_{+}-f_{-}$. We set

$$
\begin{gathered}
m_{i}^{-}(u):=\delta_{i}\left|u_{i}\right|+\sum_{j \neq i} \delta_{j}\left(u_{j}\right)_{-}, \quad m_{i}^{+}(u):=\delta_{i}\left|u_{i}\right|+\sum_{j \neq i} \delta_{j}\left(u_{j}\right)_{+}, \\
F_{i}^{*}(M, p, u)=\mathcal{L}^{-}(M, p)+m_{i}^{+}(u), i=1, \ldots, n .
\end{gathered}
$$

for some $\delta_{1}, \ldots, \delta_{n} \geq 0$. Further, we shall make use of the following condition, which permits to compare operators. Given two operators $F(M, p, u, x)$, $H(M, p, u, x)$, we say that $H$ satisfies condition $\left(D_{F}\right)$ provided

$$
\begin{aligned}
-F(N-M, q-p, v-u, x) & \leq H(M, p, u, x)-H(N, q, v, x) \\
& \leq F(M-N, p-q, u-v, x) .
\end{aligned}
$$

We shall use this condition for operators $F$ which are convex in $(M, p, u)$ (this is equivalent to supposing that $F$ itself satisfies $\left(D_{F}\right)$ ). Note $F_{i}^{*}$ are convex. In general, HJB operators are convex. 
We suppose that the operators $H_{i}$ are uniformly elliptic with bounded measurable coefficients, that is, for some $\gamma, \delta>0$ and all $M, N \in \mathcal{S}_{N}, p, q \in$ $\mathbb{R}^{N}, u, v \in \mathbb{R}^{n}, x \in \Omega$,

$\left(H_{1}\right) \quad H_{i}$ satisfies $\left(D_{F_{i}^{*}}\right), i=1, \ldots, n$

Note the definition of $m_{i}$ and $\left(H_{1}\right)$ imply that the function $H_{i}(M, p, u, x)$ is nondecreasing in all variables $u_{j}$ with $j \neq i$. Such a system is called quasimonotone.

Since we want to prove existence results, we are going to suppose that $\left(H_{2}\right)$

$$
H_{i}(M, 0,0, x) \text { is continuous in } \mathcal{S}_{N} \times \bar{\Omega} \text {. }
$$

Viewing to get results on existence of eigenvalues, we are led to suppose that these operators are positively homogeneous.

$$
H_{i}(t M, t p, t u, x)=t H_{i}(M, p, u, x), \quad \text { for all } t \geq 0, i=1, \ldots, n .
$$

In order to obtain precise results, it will be important to describe the coupling in the system, that is, the way it relates the functions $u_{i}$ to each other. It turns out that this description can be done in terms of the bounded measurable functions $c_{i j}(x):=F_{i}\left(0,0, e_{j}, x\right)$, where $e_{j} \in \mathbb{R}^{n}$ is the vector whose $j$-th coordinate is 1 and all other coordinates are zero. We set $\mathcal{C}(x):=$ $\left(c_{i j}(x)\right)_{i, j=1}^{n}$. As explained in [BS], any such matrix can have its lines and columns renumbered in such a way that it is in block triangular form, with each block on the main diagonal being irreducible. We recall a $n \times n$ matrix $\mathcal{C}$ is called irreducible provided for any non-empty sets $I, J \subset\{1, \ldots, n\}$ such that $I \cap J=\emptyset$ and $I \cup J=\{1, \ldots, n\}$, there exist $i_{0} \in I$ and $j_{0} \in J$ for which

$$
\operatorname{meas}\left\{x \in \Omega \mid c_{i_{0} j_{0}}(x)>0\right\}>0 \text {. }
$$

For simplicity, when (3) holds we write $c_{i_{0} j_{0}} \not \equiv 0$ in $\Omega$. Whenever $\mathcal{C}$ is irreducible, we will say that (1) is fully coupled. Simply speaking, a system is fully coupled provided it cannot be split into two subsystems, one of which does not depend on the other.

More precisely, we can always renumber the equations in (1) and the components of $u$ in such a way that we can write $\mathcal{C}=\left(\mathcal{C}_{k l}\right)_{k, l=1}^{m}$, where $1 \leq m \leq n, \mathcal{C}_{k l}$ are $t_{k} \times t_{l}$ matrices for some $t_{k} \leq n$ with $\sum_{k=1}^{m} t_{k}=n, \mathcal{C}_{k k}$ is an irreducible matrix for all $k=1, \ldots, m$, and $\mathcal{C}_{k l} \equiv 0$ in $\Omega$, for all $k, l \in$ $\{1, \ldots, m\}$ with $k<l$. Note that $m=1$ means $\mathcal{C}$ itself is irreducible, while $m=n$ means $\mathcal{C}$ is in triangular form. We set $s_{0}=0, s_{k}=\sum_{j=1}^{k} t_{j}$, and $S_{k}=\left\{s_{k-1}+1, \ldots, s_{k}\right\}$.

For instance, any $1 \times 1$ matrix is irreducible. Up to renumbering, when $n=2$ we divide the set of $2 \times 2$ matrices $\mathcal{C}$ into two parts : matrices of 
the form $\left(\begin{array}{ll}* & a \\ b & *\end{array}\right)$ and matrices of the form $\left(\begin{array}{ll}* & 0 \\ * & *\end{array}\right)$, where $a, b \neq \equiv 0$ and $*$ stands for an arbitrary function. The first of these matrices is irreducible, the second is not. Respectively, for $n=3$, there are four types of matrices (of course, up to renumbering again) :

$$
\left(\begin{array}{lll}
* & a & * \\
* & * & b \\
c & * & *
\end{array}\right), \quad\left(\begin{array}{lll}
* & a & 0 \\
b & * & c \\
0 & d & *
\end{array}\right), \quad\left(\begin{array}{ccc}
* & a & 0 \\
b & * & 0 \\
* & * & *
\end{array}\right), \quad\left(\begin{array}{ccc}
* & 0 & 0 \\
* & * & 0 \\
* & * & *
\end{array}\right), \quad a, b, c, d \not \equiv 0 .
$$

The first two of these matrices are irreducible, the third has one $2 \times 2$ and one $1 \times 1$ irreducible blocks, and the fourth has three $1 \times 1$ irreducible blocks.

We make the convention that all (in)equalities between vectors will be understood to hold component-wise. Also, any time we speak of a solution (supersolution, subsolution) we shall mean $L^{N}$-viscosity solutions, unless otherwise stated. We refer to [CCKS] and [BS] for a general review of $L^{N}$-viscosity solutions. Note that viscosity solutions are continuous and that any function in $W_{\text {loc }}^{2, N}(\Omega)$ satisfies (1) almost everywhere - such a solution is called strong - if and only if it is a $L^{N}$-viscosity solution.

In the following we denote with $\mathcal{F}[\psi]$ the vector $\left(F_{i}\left(D^{2} \psi_{i}, \psi_{i}, \psi, x\right)\right)_{i=1}^{n}$, for any vector function $\psi$, respectively we denote with $\mathcal{H}[\psi]$ the vector $\left(H_{i}\left(D^{2} \psi_{i}, \psi_{i}, \psi, x\right)\right)_{i=1}^{n}$.

Our first result concerns existence of eigenvalues for fully coupled systems. We set

$$
\begin{aligned}
& \lambda_{1}^{+}=\lambda_{1}^{+}(\mathcal{F}, \Omega)=\sup \left\{\lambda \in \mathbb{R} \quad \mid \quad \text { there exists } \psi \in C\left(\Omega, \mathbb{R}^{n}\right)\right. \text { such that } \\
& \psi>0 \text { and } \mathcal{F}[\psi]+\lambda \psi \leq 0 \text { in } \Omega\} \text {. } \\
& \lambda_{1}^{-}=\lambda_{1}^{-}(\mathcal{F}, \Omega)=\sup \left\{\lambda \in \mathbb{R} \quad \mid \quad \text { there exists } \psi \in C\left(\Omega, \mathbb{R}^{n}\right)\right. \text { such that } \\
& \psi<0 \text { and } \mathcal{F}[\psi]+\lambda \psi \geq 0 \text { in } \Omega\} \text {. }
\end{aligned}
$$

Theorem 1 Suppose that $F_{i}(M, p, u, x)$ satisfies $\left(H_{0}\right)-\left(H_{2}\right)$ and is convex in $(M, p, u)$, for all $1 \leq i \leq n$. Assume $\mathcal{C}=\left(F_{i}\left(0,0, e_{j}, x\right)\right)_{i, j=1}^{n}$ is an irreducible matrix. Then

(a) there exists vectors $\varphi_{1}^{+}, \varphi_{1}^{-} \in W_{\text {loc }}^{2, q}\left(\Omega, \mathbb{R}^{n}\right) \cap C\left(\bar{\Omega}, \mathbb{R}^{n}\right), \forall q<\infty$, such that

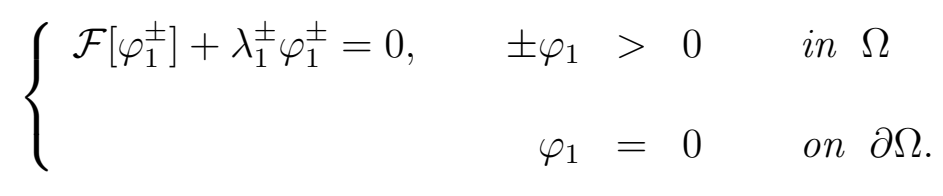


(b) we have $\lambda_{1}^{+} \leq \lambda_{1}^{-}$and $\lambda_{1}^{+}$can be the only eigenvalue of $\mathcal{F}$ in the interval $\left(-\infty, \lambda_{1}^{-}\right)$. If $\lambda_{1}^{+}=\lambda_{1}^{-}$then $\varphi_{1}^{+} \equiv \varphi_{1}^{-} ;$the vector $\varphi_{1}^{ \pm}$spans $\operatorname{Ker}\left(\mathcal{F}+\lambda_{1}^{ \pm}\right)$in $C\left(\bar{\Omega}, \mathbb{R}^{n}\right)$ under the Dirichlet boundary condition.

(c) assume there is a vector $\Psi \in C\left(\Omega, \mathbb{R}^{n}\right)$ such that $\Psi>0$ and $\mathcal{F}[\Psi] \leq 0$ in $\Omega$. Then either $\lambda_{1}^{+}>0$ or $\lambda_{1}^{+}=0$ and $\Psi=$ const. $\varphi_{1}^{+}$.

(d) if we normalize $\varphi_{1}^{ \pm}=\left(\phi_{1,1}^{ \pm}, \ldots, \phi_{1, n}^{ \pm}\right)$in such a way that $\min _{1 \leq i \leq n} \phi_{1, i}^{ \pm}\left(x_{0}\right)=1$ for some $x_{0} \in \Omega$, then

$$
\sup _{\Omega} \varphi_{1}^{ \pm}:=\sup _{\Omega} \max _{1 \leq i \leq n} \phi_{1, i}^{ \pm} \leq C,
$$

where $C$ depends only on $x_{0}, \Omega, \lambda, \Lambda, \gamma, \delta$, and a lower bound on the measures of the subsets of $\Omega$ on which $c_{i j}>0$, where $c_{i j}$ are the functions used to define the full coupling in $\mathcal{C}$.

It follows from this theorem that to any $\mathcal{F}$ such that $F_{i}(M, p, u, x)$ satisfies $\left(H_{0}\right)-\left(H_{2}\right)$ and is convex in $(M, p, u)$ we can associate a set of numbers $\lambda_{11}^{ \pm}, \ldots, \lambda_{1 m}^{ \pm}$, where $m$ is the number of irreducible blocks which appear in the decomposition of $\mathcal{C}$, and $\lambda_{1 k}^{ \pm}$are the eigenvalues, given by Theorem 1 , of the subsystem containing only the operators $\left(F_{i}\right)$ with indices $i \in S_{k}$, in which all $u_{j}, j \notin S_{k}$, are set to zero. We denote

$$
\lambda_{1}^{ \pm}:=\min _{1 \leq k \leq m} \lambda_{1 k}^{ \pm}
$$

The positivity of the eigenvalues turns out to be a necessary and sufficient condition for the vector operator $\mathcal{F}$ to satisfy the comparison principle and the Alexandrov-Bakelman-Pucci inequality, and to be a sufficient condition for the Dirichlet problem (1) to be solvable.

We recall that a second order operator $\mathcal{H}$ satisfies the comparison principle $(\mathrm{CP})$, provided for any $u, v \in C\left(\bar{\Omega}, \mathbb{R}^{n}\right)$, one of which is in $W_{\text {loc }}^{2, N}(\Omega)$, such that

$$
\left\{\begin{array}{cll}
\mathcal{H}[u] & \geq \mathcal{H}[v] & \text { in } \quad \Omega \\
u & \leq v & \text { on }
\end{array}\right.
$$

we have $u \leq v$ in $\Omega$. A particular case of $(\mathrm{CP})$ is the maximum principle, when one of $u, v$ is set to zero (and $H(0,0,0, x) \equiv 0$, as we always assume).

Theorem 2 Suppose a second-order operator $\mathcal{F}$ is such that $F_{i}(M, p, u, x)$ satisfies $\left(H_{0}\right)-\left(H_{2}\right)$ and is convex in $(M, p, u)$, for all $1 \leq i \leq n$. Then $\lambda_{1}^{+}(\mathcal{F})>0$ is necessary and sufficient for $\mathcal{F}$ to satisfy $(C P)$.

Hence, if a second-order operator $\mathcal{H}$ satisfies $\left(D_{\mathcal{F}}\right)$, then $\lambda_{1}^{+}(\mathcal{F})>0$ is sufficient for $H$ to satisfy $(C P)$. 
Theorem 3 Suppose the second-order operator $\mathcal{F}$ is such that $F_{i}(M, p, u, x)$ satisfies $\left(H_{0}\right)-\left(H_{2}\right)$ and is convex in $(M, p, u)$, for all $1 \leq i \leq n$.

I. If $\lambda_{1}^{-}(\mathcal{F})>0$ then for any $u \in C(\bar{\Omega}, \mathbb{R}), f \in L^{N}(\Omega, \mathbb{R})$, the inequality $\mathcal{F}[u] \leq f$ implies

$$
\sup _{\Omega} \max _{1 \leq i \leq n}\left(u_{i}\right)_{-} \leq C\left(\sup _{\partial \Omega} \max _{1 \leq i \leq n}\left(u_{i}\right)_{-}+\left\|\max _{1 \leq i \leq n}\left(f_{i}\right)_{+}\right\|_{L^{N}(\Omega)}\right)
$$

where $C$ depends on $\Omega, N, \lambda, \Lambda, \gamma, \delta$, and $\lambda_{1}^{-}(\mathcal{F})$.

II. In addition, if $\lambda_{1}^{+}(\mathcal{F})>0$ then $\mathcal{F}[u] \geq f$ implies

$$
\sup _{\Omega} \max _{1 \leq i \leq n} u_{i} \leq C\left(\sup _{\partial \Omega} \max _{1 \leq i \leq n}\left(u_{i}\right)_{+}+\left\|\max _{1 \leq i \leq n}\left(f_{i}\right)_{+}\right\|_{L^{N}(\Omega)}\right) \text {. }
$$

We have the following general result on solvability of (1).

Theorem 4 Suppose the second-order operator $\mathcal{F}$ is such that $F_{i}(M, p, u, x)$ satisfies $\left(H_{0}\right)-\left(H_{2}\right)$ and is convex in $(M, p, u)$, for all $1 \leq i \leq n$, and suppose that that the second-order operator $\mathcal{H}$ satisfies $\left(D_{\mathcal{F}}\right)$ (recall that $\mathcal{F}$ always satisfies $\left(D_{\mathcal{F}}\right)$ ).

I. If $\lambda_{1}^{+}(\mathcal{F})>0$ then for any $f \in L^{p}\left(\Omega, \mathbb{R}^{n}\right), p \geq N$, there exists a viscosity solution $u \in C\left(\bar{\Omega}, \mathbb{R}^{n}\right)$ of

$$
\begin{aligned}
& \mathcal{H}[u]=f \text { in } \Omega \\
& u=0 \text { on } \partial \Omega \text {. }
\end{aligned}
$$

If in addition all $H_{i}(M, p, u, x)$ are convex in $M$ then $u$ is the unique viscosity solution of this problem, and $u \in W_{\text {loc }}^{2, p}\left(\Omega, \mathbb{R}^{n}\right), p<\infty$.

II. If we only know that $\lambda_{1}^{-}(\mathcal{F})>0$ then for any $f \in L^{p}\left(\Omega, \mathbb{R}^{n}\right), p \geq N$, such that $f \geq 0$ in $\Omega$, there exists a solution $u \in W_{\text {loc }}^{2, p}\left(\Omega, \mathbb{R}^{n}\right) \cap C\left(\bar{\Omega}, \mathbb{R}^{n}\right)$, such that $u \leq 0$ in $\Omega$, of

$$
\begin{aligned}
& \mathcal{F}[u]=f \text { in } \Omega \\
& u=0 \text { on } \partial \Omega \text {. }
\end{aligned}
$$

Note that we could always take $\mathcal{F}$ in the last theorem to be the extremal operator from $\left(H_{1}\right)$, if $\lambda_{1}^{+}\left(\mathcal{F}^{*}\right)>0$; however using $\left(D_{\mathcal{F}}\right)$ gives a more precise result, in the sense that we may have $\lambda_{1}^{+}\left(\mathcal{F}^{*}\right) \leq 0$ but $\lambda_{1}^{+}(\mathcal{F})>0$ for some "intermediate" $\mathcal{F}$ between $\mathcal{H}$ and $\mathcal{F}^{*}$.

It is important for applications to have bounds on the eigenvalues in terms of the operator and the domain. These bounds permit to verify the hypotheses of the previous theorems. 
Theorem 5 Suppose the second-order operator $\mathcal{F}$ is such that $F_{i}(M, p, u, x)$ satisfies $\left(H_{0}\right)-\left(H_{2}\right)$ and is convex in $(M, p, u)$, for all $1 \leq i \leq n$. Set $\delta=\sum_{i=1}^{n} \delta_{i}$, where $\delta_{i}$ are the numbers from $\left(H_{1}\right)$.

Let $d$ and $|\Omega|$ denote respectively the diameter and the Lebesgue measure of $\Omega$, and let $R$ be the radius of the largest ball inscribed in $\Omega$. There exists a positive constant $C_{1}$, which depends only on $N, n, \lambda, \Lambda, \gamma, d$, and a positive constant $C_{2}$, which depends only on $N, n, \lambda, \Lambda, \gamma\left(C_{1}, C_{2}\right.$ are bounded when these quantities are bounded), such that

$$
\frac{1}{C_{1} d|\Omega|^{1 / N}}-\delta \leq \lambda_{1}^{+}(\mathcal{F}) \leq \lambda_{1}^{-}(\mathcal{F}) \leq \frac{C_{2}(1+\delta)}{R^{2}}
$$

\section{References}

[BS] J. Busca, B. Sirakov, Harnack type estimates for nonlinear elliptic systems and applications, Ann. Inst. H. Poincare, Anal. Nonl. 21 (5) (2004), 543-590.

[CCKS] L.A. Caffarelli, M.G. Crandall, M.Kocan, A. Świech, On viscosity solutions of fully nonlinear equations with measurable ingredients, Comm. Pure Appl. Math. 49 (1996), 365-397.

[IK] H. Ishii, S. Koike, Viscosity solutions for monotone systems of secondorder elliptic PDE's, Comm. Part. DIff. Eq. 16(6-7) (1991), 1095-1128.

[QS] A. Quaas, B. Sirakov, Principal eigenvalues and the Dirichlet problem for fully nonlinear elliptic operators, to appear ; Comptes Rendus Acad. Sc. Ser. I, 342 (2006), 115-118. 